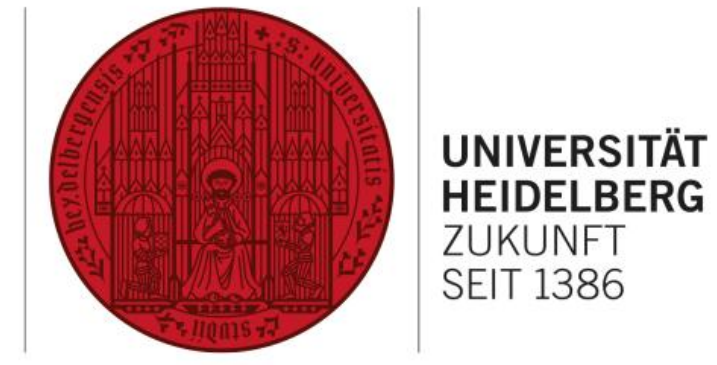

Zusammenfassung der Dissertation mit dem Titel

\title{
„Zwischen Religion und Selbstbestimmung. Karitative Tätigkeit der Religionsgemeinschaften vor neuen Herausforderungen anlässlich der gesetzlichen Regelung zur Patientenverfügung“"
}

Dissertation vorgelegt von Franziska Buchwald

Erstgutachter: Prof. Dr. Bernd Grzeszick

Zweitgutachter: Prof. Dr. Peter Axer

Institut für Staatsrecht, Verfassungslehre und Rechtsphilosophie 


\section{Zwischen Religion und Selbstbestimmung.}

Karitative Tätigkeit der Religionsgemeinschaften vor neuen Herausforderungen anlässlich der gesetzlichen Regelung zur Patientenverfügung

Kurzzusammenfassung der Dissertation von Franziska M. Buchwald 2012

Die Dissertation beschäftigt sich mit dem erst im Jahr 2009 gesetzlich verankerten Instrument zur Vorsorge in Gesundheitsangelegenheiten, der Patientenverfügung. Dabei werden nicht die rechtsphilosophischen Hintergründe beleuchtet oder die rechtlichen Umsetzungsmöglichkeiten betrachtet, vielmehr werden die Herausforderungen und Handlungsmöglichkeiten aufgezeigt, vor denen die karitative Tätigkeit der Religionsgemeinschaften anlässlich der gesetzlichen Regelung der Patientenverfügung heute steht. Damit nimmt die Arbeit eine in die Zukunft gerichtete Perspektive ein, was auch daran deutlich wird, dass die in ihr behandelte Problematik mittlerweile erkannt und auch öffentlich diskutiert wird.

Einige Religionsgemeinschaften, insbesondere die katholische Kirche, sind der Ansicht, dass der Mensch nicht über das Leben verfügen dürfe. Von diesem Standpunkt aus, ist der im Voraus verfügte Wille eines Patienten, dass lebensverlängernde Maßnahmen an ihm nicht vorgenommen werden sollen, nur dann beachtlich, wenn der Sterbeprozess bereits begonnen hat. Bei einem Demenzkranken im fortgeschrittenen Stadium oder einem Wachkomapatient hat dieser Sterbeprozess medizinisch grundsätzlich noch nicht begonnen. Nach Ansicht der katholischen Kirche kann der Wille des Patienten, sein Leben beenden zu lassen und keine lebensverlängernden Behandlungsmaßnahmen mehr zu ergreifen, dann nicht verbindlich sein. Der Standpunkt des Gesetzgebers ist ein anderer. Der Bundestag hat nach jahrelanger Diskussion um eine gesetzliche Regelung zur Patientenverfügung die Reichweitenklarstellung in § 1901a Abs. 3 BGB erlassen, nach dem der Wille des Patienten „unabhängig von Art und Stadium der Erkrankung“ gilt.

Kurz nachdem das Gesetz im September 2009 in Kraft getreten war, aktualisierte der Bischof der Diözese Stuttgart-Rottenburg Gebhard Fürst die bereits im Laufe des Gesetzgebungsprozesses von der Kirche geäußerte Kritik an der Verbindlichkeit des Patientenwillens unabhängig davon, auf welche Art von Krankheit und auf welches Stadium er sich bezieht und erklärte: „Im Hinblick auf den Aspekt der Reichweite kommt für uns als Kirchen aufgrund unseres Menschenbildes nach wie vor eine solche Verfügung nicht zum 
Tragen, wenn der Tod nicht unmittelbar bevorsteht, z.B. bei anhaltendem Koma und fortgeschrittener Demenzerkrankung.“

Angesichts dieser unterschiedlichen Wertung hinsichtlich des Rechts auf Selbstbestimmung über den eigenen Körper bis an das Lebensende stellt sich die Frage, ob und wie dieser Konflikt zwischen klarer gesetzlicher Anordnung und theologischer Ablehnung zu lösen ist. Angesichts der Vielzahl von Menschen, die in karitativen Kranken- und Pflegeeinrichtungen versorgt und von Mitarbeitern karitativer Betreuungsvereine betreut werden und die zunehmend nach Selbstbestimmung bis in den Tod streben, ist die Klärung dieser Frage nicht rein akademischer Natur. Es ist zu fragen, ob ein Demenzkranker bei einer Versorgung in einer karitativen Einrichtung Gefahr läuft, dass sein Wille missachtet wird. Es stehen sich in diesem Konflikt Religionsgemeinschaften, karitative Einrichtungen, Patienten sowie der Staat gegenüber. Die grundrechtlichen Freiheitsrechte sind hier bedeutsam. Der Patient kann sich auf sein Recht auf körperliche Unversehrtheit und Selbstbestimmung über seinen Körper berufen, unabhängig davon ob man dies nun allein in Art. 2 Abs. 2 GG oder in Art. 2 Abs. 1 GG, ggf. i.V.m. Art. 1 Abs. 1 GG verortet; den Religionsgemeinschaften steht das Recht auf Bestimmung ihrer eigenen Angelegenheiten aus Art. 140 GG i.V.m. Art. 137 Abs. 3 S. 1 WRV ebenso wie das Recht auf Religionsfreiheit aus Art. 4 Abs. 1, 2 GG zu; auch die karitativen Einrichtungen können sich auf diese beiden spezifischen Freiheitsrechte berufen. Der Konflikt steht zwischen Religion und Selbstbestimmung.

In der Arbeit wird zunächst das Spannungsfeld zwischen dem sozialen Staat und der karitativen Kirche im Abstrakten sowie die Wertungsdifferenz zwischen Staat und Religionsgemeinschaften im Bezug auf das Ende des Lebens im Konkreten dargestellt. Dazu erfolgt auch eine Untersuchung der Regelungsinhalte von § 1901a BGB. Anschließend wird der freiheitliche Gestaltungsraum für die karitative Betätigung von religiösen Einrichtungen untersucht, wobei zu beachten war, dass der Freiheitsraum nicht unantastbar ist. Vielmehr kann er durch verfassungsmäßige Gesetze beschränkt werden, wobei der Verhältnismäßigkeitsgrundsatz fordert, dass Gestaltungsmöglichkeiten verbleiben, die dazu beitragen, die religiösen Vorstellungen zu sichern und damit zu verhindern, dass die Religionsgemeinschaften ihrem Auftrag und ihrer Lehre zuwider handeln müssten. 
Das Aufzeigen und die Diskussion dieser Gestaltungsmöglichkeiten stehen im Mittelpunkt der Arbeit. Dadurch soll der Rechtspraxis eine Hilfestellung gegeben werden.

Zum einen wird die (wenn auch eher theoretische) Möglichkeit des Rückzugs der karitativen Einrichtungen aus der professionellen Krankenversorgung und Pflege sowie Betreuung erörtert. Ein Rückzug wäre unter Wahrung der sozialgesetzlichen Kündigungsfristen einfachrechtlich und verfassungsrechtlich zulässig. Mit dieser Lösung wären jedoch für Staat, Gesellschaft und Religionsgemeinschaften eine Vielzahl an Nachteilen, auch nichtjuristischer Art, verbunden.

Die schlichte Fortführung der karitativen Arbeit unter Beachtung der gesetzlichen Vorgaben ohne besondere Vorkehrungen zur Wahrung religiöser Vorstellungen hingegen würde zu einer teilweisen Selbstsäkularisation führen und könnte über kurz oder lang zu einer Veränderung der Privilegien der Religionsgemeinschaften führen.

Ein Recht, diejenigen Handlungen zu verweigern, die im Widerspruch zur religiösen Vorstellung stehen, lässt sich in Bezug auf das Lebensende weder dem einfachen Gesetz, noch dem Verfassungsrecht direkt entnehmen. Entsprechend hat auch der BGH im Juni 2005 entschieden, als er beurteilen musste, ob das Pflegeheim dem Verlangen des Betreuers, die künstliche Ernährung bei einem Wachkomapatienten einzustellen, nachkommen muss. Das Personal einer Einrichtung ist grundsätzlich verpflichtet, den Willen des Patienten zu beachten und kann einen Behandlungsabbruch insofern nicht verweigern. Der Gesetzgeber hat bei der gesetzlichen Regelung der Patientenverfügung (anders als in § 12 SchKG) davon abgesehen, ein Weigerungsrecht einzuräumen.

Es bietet sich allerdings an, die Verträge zwischen dem Benutzer (dem Patienten) und dem Träger einer Kranken- und Pflegeeinrichtung (sogenannter Pflegeheimvertrag und Krankenhausaufnahmevertrag) zu gestalten, so dass Konflikte bereits von vornherein vermieden werden. Bei der Vertragsgestaltung sind eine Reihe gesetzlicher Vorgaben zu beachten, insbesondere das Kopplungsverbot aus § 1901a BGB, die sozialrechtlichen Vorgaben, die Bestimmungen des Wohn- und Betreuungsvertragsgesetzes sowie das Recht über Allgemeine Geschäftsbedingungen. Wegen des Kopplungsverbots scheiden Lösungsansätze aus, die Patienten vertraglich dazu verpflichten, eine religionskonforme Patientenverfügung zu verfassen oder das Vorliegen einer solchen zur Bedingung zu machen. Ein heimvertraglicher Leistungsausschluss dahingehend, dass Versorgungs- und Behand- 
lungsmaßnahmen ausgeschlossen werden, die einen Behandlungsabbruch außerhalb der Sterbephase begleiten oder ermöglichen sollen, ist aber rechtlich möglich. Dies gilt jedoch nur dann, wenn besondere Vorkehrungen beachtet werden, die allesamt Eingang in den Vertrag finden müssen. Zudem muss eine Abstimmung mit den sozialrechtlichen Verträgen erfolgen. Wegen des engmaschigen Regelungssystems im Krankenhauswesen ist dieser Weg bei dem Abschluss eines Krankenhaushausaufnahmevertrags allerdings nicht gangbar.

Neben der Krankenversorgung und der Pflege engagieren sich religiöse Träger im Bereich der Betreuung. Durch die bereits bestehende harmonisierende Rechtslage steht für die karitative Arbeit dort aber zunächst hinreichend Raum zur Verfügung, um den religiösen Vorstellungen nicht zuwider handeln zu müssen. Weiterer Handlungsbedarf besteht insofern nicht.

Die Religionsgemeinschaften können gemäß Art. 140 GG i.V.m. 137 Abs. 3 S. 1 WRV selbst darüber bestimmen, welche Einrichtungen zu ihr gehören sollen. Ihnen obliegt es damit, selbst über die Zugehörigkeit einer Einrichtung beziehungsweise die Aberkennung der Zugehörigkeit zu entscheiden. Sie können die Entscheidung davon abhängig machen, dass die Einrichtungen von den Gestaltungsmöglichkeiten Gebrauch machen, indem dies zur Zugehörigkeitsvoraussetzung gemacht wird. So kann die Religionsgemeinschaft die Tätigkeit von karitativen Einrichtungen selber mittelbar steuern, obwohl die Einrichtungen grundsätzlich rechtlich selbständig sind. Im katholischen Bereich kann zudem über das Kirchenrecht - can. $300 \mathrm{CIC}$ - erreicht werden, dass sich kein Verein ohne Zustimmung der Autorität „katholisch“ nennen darf.

Selbst nach den einschlägigen Entscheidungen des EGMR stellt zudem das kirchliche Arbeitsrecht weiterhin ein Instrumentarium dar, mit dem die Steuerung des in einer karitativen Einrichtung tätigen Personals erreicht werden kann. Über Leistungstreuepflichten und Loyalitätsobliegenheiten kann verhindert werden, dass das Personal in karitativen Einrichtungen den religiösen Vorstellungen zuwider handelt. Allerdings ist dafür entscheidend, wie weit der Gestaltungsraum der Einrichtungen reicht: Nur wenn eine Leistung ausgeschlossen, ein Behandlungsabbruch verweigert werden darf, kann die Einrichtung verlangen, dass der Beschäftigte diese Handlung nicht begeht. Zudem finden diese Pflichten und Obliegenheiten ihre Grenze im Strafrecht. 
Schließlich wird dargelegt, dass eine organisatorische Umgestaltung durch die Wahl einer genossenschaftlichen Rechtsform zur Problemlösung nicht in Betracht kommt. Die Diskussion der Lösungsansätze endet mit einem Hinweis auf außerrechtliche Ansätze.

Die Arbeit erscheint im Jahr 2013 bei Duncker \& Humblot, Berlin. 